\title{
Boundary Multifractality at the Integer Quantum Hall Plateau Transition: Implications for the Critical Theory
}

\author{
H. Obuse, ${ }^{1, \text { A A. R. Subramaniam, }}{ }^{2}$ A. Furusaki,${ }^{1}$ I. A. Gruzberg, ${ }^{2}$ and A. W. W. Ludwig ${ }^{3}$ \\ ${ }^{1}$ Condensed Matter Theory Laboratory, RIKEN, Wako, Saitama 351-0198, Japan \\ ${ }^{2}$ James Franck Institute and Department of Physics, University of Chicago, Chicago, IL 60637, USA \\ ${ }^{3}$ Department of Physics, University of California, Santa Barbara, CA 93106, USA
}

(Dated: August 11, 2008)

\begin{abstract}
We study multifractal spectra of critical wave functions at the integer quantum Hall plateau transition using the Chalker-Coddington network model. Our numerical results provide important new constraints which any critical theory for the transition will have to satisfy. We find a non-parabolic multifractal spectrum and determine the ratio of boundary to bulk multifractal exponents. Our results rule out an exactly parabolic spectrum that has been the centerpiece in a number of proposals for critical field theories of the transition. In addition, we demonstrate analytically exact parabolicity of related boundary spectra in the two-dimensional chiral orthogonal 'Gade-Wegner' symmetry class.
\end{abstract}

PACS numbers: 73.43.-f, 72.15.Rn, 73.20.Fz, 71.30.+h

The physics of the quantum Hall effect has been an exciting area of research for more than two decades [1, 2. While much progress has been made in this area, the identification of an analytically tractable theory describing the critical properties at the transitions between the plateaus in the integer quantum Hall (IQH) effect has been elusive ever since [3]. These quantum phase transitions are famous examples of (Anderson) localizationdelocalization (LD) transitions driven by disorder. The diverging localization length plays the role of a correlation length in non-random continuous phase transitions, known to be described by conformal field theories in two dimensions (2D). It is natural to expect that effective (field) theories describing IQH plateau transitions should generally also possess conformal symmetry (cf. 四).

Many attempts have been made in the past to identify an analytically tractable description of the IQH plateau transition and, more recently, Wess-Zumino (WZ) field theories defined on a certain supermanifold were conjectured to provide such a description [5, 6, 7. (Similar theories have also appeared in the context of string propagation in Anti-de Sitter space-time [8].) These proposals focussed solely on bulk observables, i.e., on physical quantities measured in a sample without any boundaries. In this Letter, we provide important new constraints that arise when one studies the scaling behavior of wave functions near the boundaries of a sample. Any proposed candidate theory for the plateau transitions will have to be consistent with our numerical results for the boundary multifractal spectrum.

At LD transitions, critical wave functions obey scaleinvariant, multifractal (MF) statistics, namely, disorderaveraged moments of wave functions have a power-law dependence on the linear dimension $L$ of the system [9]:

$$
\overline{|\psi(\boldsymbol{r})|^{2 q}} /\left(\overline{|\psi(\boldsymbol{r})|^{2}}\right)^{q}=C_{q}^{x}(L) L^{-\Delta_{q}^{x}} .
$$

The MF exponents $\Delta_{q}^{x}$, which are related to ('anomalous') scaling dimensions of certain operators in an un- derlying field theory [10], can be defined for points $\boldsymbol{r}$ in the bulk $(x=b)$ of the sample, $\Delta_{q}^{b}$, or near its boundary ('surface': $x=s$ ) 四, 11], $\Delta_{q}^{s}$. The prefactor $C_{q}^{x}(L)$ in Eq. (11) depends on $q$ and, in general, on $L$ if we include the possibility of corrections to scaling. Both sets of MF exponents satisfy the symmetry relation 12

$$
\Delta_{q}^{x}=\Delta_{1-q}^{x}
$$

(in some interval [4] around $q=1 / 2$ ).

Equivalently, the MF wave functions can be characterized by the so-called singularity spectra $f^{x}\left(\alpha^{x}\right)$ related to $\Delta_{q}^{x}$ by a Legendre transform: $f^{x}\left(\alpha_{q}^{x}\right)=\left(\alpha_{q}^{x}-2\right) q-$ $\Delta_{q}^{x}+d_{x}, \alpha_{q}^{x}-2=d \Delta_{q}^{x} / d q$, and $d_{b}=2, d_{s}=1$. The exponent $\alpha_{0}^{x}$ describes the scaling of typical wave functions: $\overline{\ln |\psi(\boldsymbol{r})|^{2}} \sim-\alpha_{0}^{x} \ln L$, as can be seen by taking the $q$ derivative in Eq. (1) at $q=0$.

Work emerging [13, 14] from Ref. [5] led to the conjecture that the proposed theory would give rise to an exactly parabolic bulk MF spectrum for the IQH transition

$$
\Delta_{q}^{b}=\gamma^{b} q(1-q),
$$

reminiscent of analytically obtained MF spectra for Dirac fermions in, e.g., random abelian gauge potentials 15. 16]. In those models the parabolicity of the MF spectrum can be understood through a reformulation of the problem in terms of free fields.

Previous numerical studies 13 of wave function statistics at the IQH transition appeared to exhibit a bulk MF spectrum that was indeed well described (with an accuracy of $\sim 1 \%$ ) by a parabolic fit (3) with $\gamma^{b}=$ $0.262 \pm 0.003$, seemingly providing support for the conjectures advanced in Ref. [5, 6, 7]. (In Ref. 13] the results are presented in terms of $f^{b}(\alpha)$. For a parabolic MF spectrum (3), $f^{b}\left(\alpha^{b}\right)$ is also parabolic, with a maximum at $\alpha_{0}^{b}=\gamma^{b}+2$.) 
Besides its conjectured relevance [5] to the IQH transition, the above-mentioned WZ theory is known to describe transport properties of a disordered electronic system in a different universality class [17, 18] (the chiral unitary 'Gade-Wegner' class AIII of 20, 211) which possesses an additional discrete (chiral) symmetry [20, not present in microscopic models for the IQH transition. Well-known microscopic realizations of field theories in class AIII are random bipartite hopping models, and certain network models [17, 18, 19]. The theory possesses a line of fixed points, with continuously varying critical properties parametrized by the critical longitudinal DC conductivity. (It was argued in Ref. [5] that for a particular value of this continuous parameter the WZ theory would provide a description of the IQH transition.)

In this paper we obtain two kinds of results. First, we provide results of extensive numerical work on the MF exponents at the IQH transition both at a boundary $\left(\Delta_{q}^{s}\right)$ and in the bulk $\left(\Delta_{q}^{b}\right)$. Based on these numerical results quadratic behavior in $q$ is ruled out for both quantities. Deviations from the parabolic form (3) are found to be much larger in the MF exponents $\Delta_{q}^{s}$ at a boundary. Here it is important to note that in complete analogy to the bulk, the above conjectures would also yield a quadratic dependence on $q$ of the boundary MF exponents $\Delta_{q}^{s}$. We further determine the ratio $\Delta_{q}^{s} / \Delta_{q}^{b}$ over a range of $q$. Accounting for this ratio is an important constraint on any proposed critical theory for the transition.

Secondly, we demonstrate analytically the exact parabolicity of boundary spectra, not for the chiral unitary class AIII, but for the related time-reversal invariant version, the chiral orthogonal 'Gade-Wegner' class BDI [17, 18, 19, 20]. We expect such parabolicity to also hold in the chiral unitary symmetry class.

We begin with the numerical part. Here, we study the multifractality of critical wave functions in a way similar to Ref. [13], with the goal of numerically determining the rescaled anomalous exponents

$$
\gamma_{q}^{x}=\Delta_{q}^{x} / q(1-q)
$$

both for $x=s$ (boundary) and $x=b$ (bulk).

For the case of boundary exponents we consider the critical Chalker-Coddington network model (CCNM) 22] with $4 L^{2}$ links placed on a cylinder. The dynamics of wave functions on links of the network is governed by a unitary evolution operator $U$. For each disorder realization, we numerically diagonalize $U$ and retain one critical wave function whose eigenvalue is closest to 1 . The largest system size we studied was $L=180$, and the ensemble average was taken over $3 \times 10^{5}$ samples for $L=50,60,5 \times 10^{5}$ samples for $L=80$, and $2 \times 10^{5}$ samples for $L=120,180$.

We obtain the anomalous dimensions $\Delta_{q}^{s}$ from Eq. (11). The boundary wave function coarse-grained over each plaquette along the boundary, is substituted into

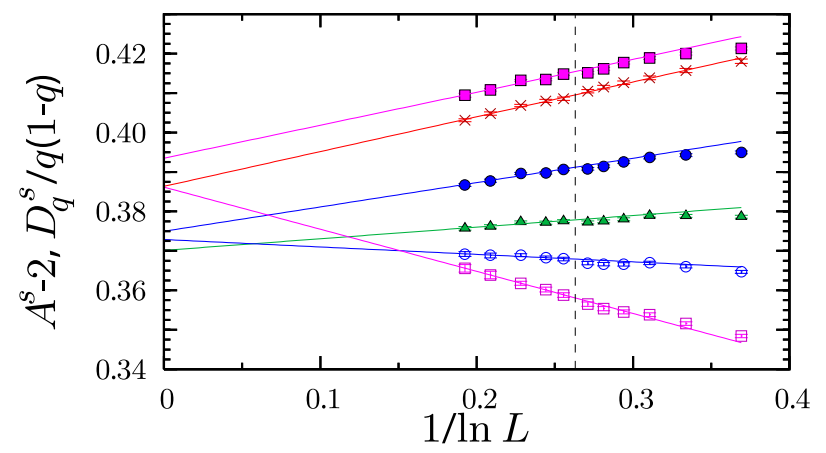

FIG. 1: (Color online) System size dependence of $D_{q}^{s}(L) / q(1-q)$ defined in the main text for $q=-0.2(\mathbf{\square})$, $0.2(\bullet), 0.5(\boldsymbol{\Delta}), 0.8(\circ)$, and $1.2(\square) . \quad \gamma_{q}^{s}$ is calculated by linear fitting taking into account only for larger system sizes $(L \geqslant 50)$ indicated by the vertical dashed line between $L=40$ and 50. We also show $A^{s}-2$ defined in the main text $(\times)$.

the left-hand side of Eq. (11), where the overline denotes ensemble and spatial averages along the boundary. Taking the logarithm, we numerically obtain $D_{q}^{s}(L) \equiv$ $\left(q \ln \overline{|\psi(\boldsymbol{r})|^{2}}-\ln \overline{|\psi(\boldsymbol{r})|^{2 q}}\right) / \ln L=\Delta_{q}^{s}-\ln C_{q}^{s}(L) / \ln L$, and plot this quantity as a function of $1 / \ln L$ in Fig. 1. We see that corrections to scaling are significant for small systems $(L \lesssim 30)$. Therefore, we used our numerical data for $L \geqslant 50$ only to extract $\gamma_{q}^{s}$ by linear fitting.

Independently, we numerically obtain $\alpha_{q}^{s}$ and $f^{s}$ from $\overline{|\psi(\boldsymbol{r})|^{2 q} \ln |\psi(\boldsymbol{r})|^{2}} / \overline{|\psi(\boldsymbol{r})|^{2 q}} \sim-\alpha_{q}^{s} \ln L$, and $\ln \overline{|\psi(\boldsymbol{r})|^{2 q}} \sim$ $\left[f^{s}\left(\alpha_{q}^{s}\right)-\alpha_{q}^{s} q-d_{s}\right] \ln L$, using our numerical data for $L \geqslant 50$. For example, the exponent $\alpha_{0}^{s}$ is obtained by linear fitting (Fig. 11), $A^{s} \equiv-\overline{\ln |\psi(\boldsymbol{r})|^{2}} / \ln L=$ $\alpha_{0}^{s}+$ const. $/ \ln L$, which yields $\alpha_{0}^{s}=2.386 \pm 0.004$.

We show in Fig. 2(a) the rescaled boundary anomalous dimension $\gamma_{q}^{s}$ (red filled circles) obtained from this analysis. We see clearly that $\gamma_{q}^{s}$ is not constant, implying that the boundary MF spectrum $\Delta_{q}^{s}$ is not parabolic. The change in $\gamma_{q}^{s}$ over the interval $0<q \leqslant 1 / 2$ is about $4 \sim 5 \%$ and is significantly larger than the error bars. This provides the strongest numerical evidence against the parabolicity of the MF exponents.

Shown in the same figure by blue open circles is the mirror image of $\gamma_{q}^{s}$ with respect to $q=1 / 2, \gamma_{1-q}^{s}$. We see that the symmetry relation (2) is satisfied within error bars for $0 \lesssim q \lesssim 1$. The rescaled anomalous dimension $\gamma_{q}^{s}$ approaches $\alpha_{0}^{s}-2$ (the horizontal line) at $q=0,1$, indicating that the two independent calculations of $\alpha_{0}^{s}$ and $\Delta_{q}^{s}$ are consistent.

We have also computed the bulk anomalous dimension $\Delta_{q}^{b}$ using the CCNM on a torus. In this case the overline in Eq. (11) implies both the ensemble and the spatial average over the whole torus. Wave functions are coarsegrained on each plaquette. We have employed the same fitting procedure as in the boundary case. The biggest system size we examined for the bulk analysis is $L=270$. The number of samples over which we took the average is $5 \times 10^{5}$ for $L=50,3 \times 10^{5}$ for $L=60,80,2 \times 10^{5}$ for 

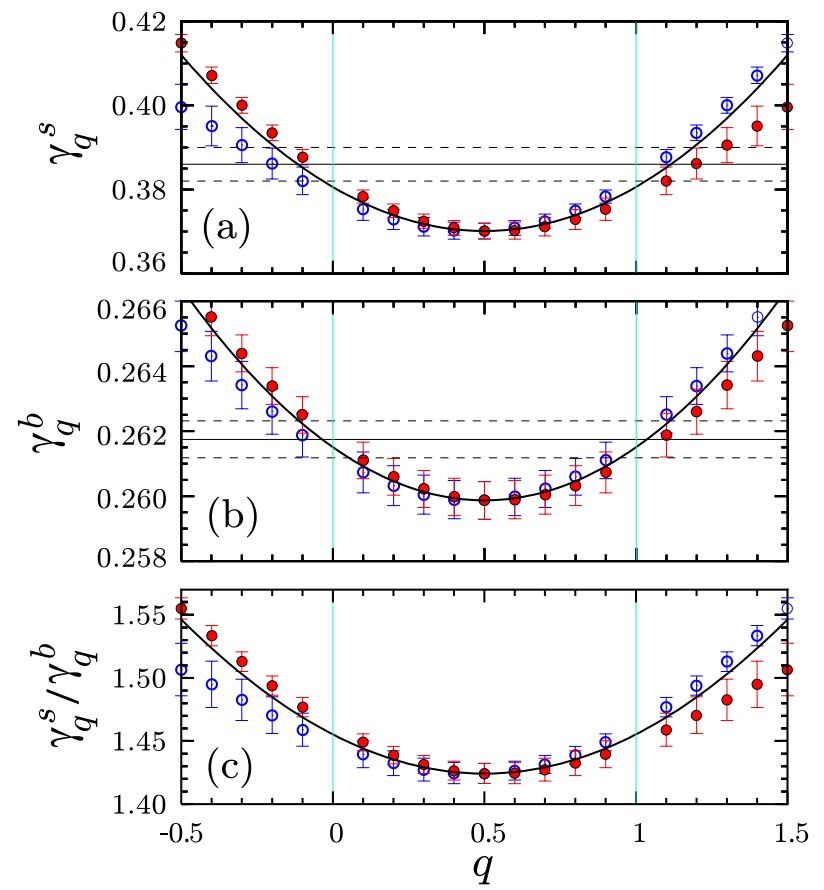

FIG. 2: (Color online) (a) Rescaled boundary MF exponents $\gamma_{q}^{s}(\bullet)$ and $\gamma_{1-q}^{s}(\circ)$. The curve is $0.370+0.042(q-1 / 2)^{2}$, obtained by fitting the data for $\gamma_{q}^{s}$ in $0<q<1$ to a parabolic form. The horizontal solid line shows $\alpha_{0}^{s}-2=0.386 \pm 0.004$ with error bars indicated by dashed lines, which is consistent with $\lim _{q \rightarrow 0,1} \gamma_{q}^{s}$. (b) Rescaled bulk MF exponents $\gamma_{q}^{b}(\bullet)$ and $\gamma_{1-q}^{b}$ (०). The curve is $0.2599+0.0065(q-1 / 2)^{2}$ obtained by fitting the data for $\gamma_{q}^{b}$ in $0<q<1$ to a parabolic form. The horizontal solid line shows $\alpha_{0}^{b}-2=0.2617 \pm 0.0006$ with error bars indicated by dashed lines. (c) Ratios $\gamma_{q}^{s} / \gamma_{q}^{b}(\bullet)$ and $\gamma_{1-q}^{s} / \gamma_{1-q}^{b}(\mathrm{o})$. As above, the curve is obtained from the parabolic fits for $\gamma_{q}^{s, b}$, which amounts to quartic approximations for $\Delta_{q}^{s, b}$.

\section{$L=120,4 \times 10^{4}$ for $L=180$, and $2 \times 10^{4}$ for $L=270$.}

Figure 2(b) shows the exponents $\gamma_{q}^{b}$, together with their mirror image. The symmetry relation (2) is again satisfied for $0 \lesssim q \lesssim 1$ within error bars, which provides confirmation that our results are reliable. We see clearly that $\gamma_{q}^{b}$ has $q$ dependence, although it is weaker than that of $\gamma_{q}^{s}$; compare the vertical scales of Fig. 2 $2(\mathrm{a})$ and (b).

The ratio $\gamma_{q}^{s} / \gamma_{q}^{b}$ is shown in Fig. 2(c) and is seen to be clearly dependent on $q$. Any candidate theory for the IQH transition needs to be consistent with this ratio, and in particular its dependence on $q$. (Note that for a free field this ratio would be equal to 2 , and independent of $q[11,23]$.)

Figure 3(a) shows $\alpha_{q}^{x}$ as a function of $q$. The data significantly deviate from linear behavior that would result if $\Delta_{q}^{x}$ were strictly parabolic (constant $\gamma_{q}^{x}$ ). In Fig. 3(b) we show the corresponding singularity spectra $f^{x}\left(\alpha_{q}^{x}\right)$ as functions of $q$. (Combining the data from the two panels would result in $f^{x}\left(\alpha^{x}\right)$ as functions of $\alpha^{x}$.) For $q \gtrsim 1.5$ where $f^{s}\left(\alpha^{s}\right)<0$, the moments $\overline{|\psi(\boldsymbol{r})|^{2 q}}$ are dominated by rare events, and thus accurate numerical calculation of

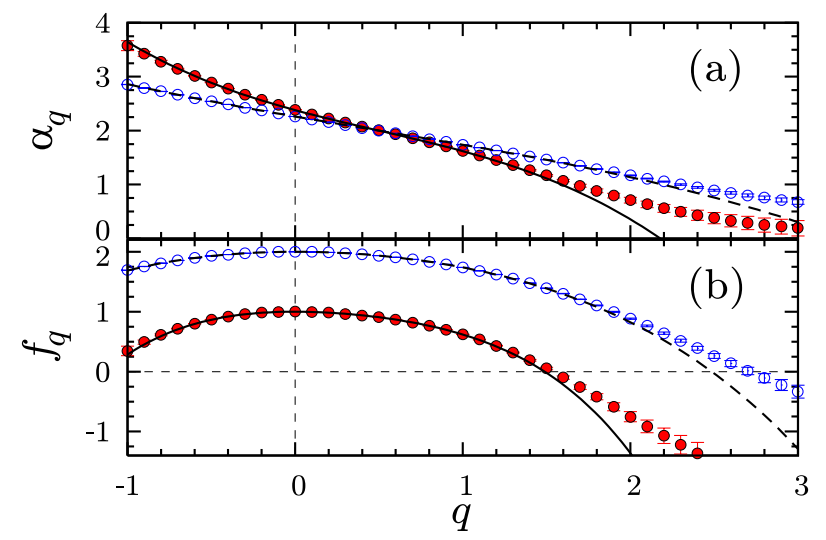

FIG. 3: (Color online) (a) $\alpha_{q}^{s}(\bullet)$ and $\alpha_{q}^{b}$ (०) as functions of $q$; (b) $f_{q}^{s}(\bullet)$ and $f_{q}^{b}(\circ)$ as functions of $q$. The solid and dashed curves on both panels are obtained from the parabolic approximations to $\gamma_{q}^{x}$ (that is, quartic approximations to $\Delta_{q}^{x}$ ). Notice that $\alpha_{q}^{x}$ significantly deviate from straight lines which would follow from strictly parabolic $\Delta_{q}^{x}$ (or constant $\gamma_{q}^{x}$ ).

MF exponents becomes more difficult [9]. This explains the discrepancy between the (red) data points and the solid curves for $q \gtrsim 1.5$ in Fig. 3. As $f_{q}^{s}>0$ at $q \gtrsim-1$, we expect that our numerical results of $f^{s}\left(\alpha^{s}\right)$ should be more reliable at $q \approx-1$ than at $q \approx 1.5$, as evidenced by the agreement between the red dots and the solid curve in Fig. 3. The curve suggests termination of $f^{s}\left(\alpha^{s}\right)$ [9] to occur at $q \approx 2.2$.

We now proceed to present our second (analytical) result. We first recall that the theory conjectured in Ref. [5] to describe the IQH plateau transition is a WZ model with global psl $(2 \mid 2)$ (super-) symmetry. This theory possesses two coupling constants, one denoted by $f$ for the kinetic term and another denoted by $k$ for the WZ term $(k=1$ in Ref. [5]), in standard notation [5, 24]. One can think of this theory as a perturbation of the rather well understood [24, 25] Kac-Moody (KM) point characterized by the condition $f^{-2}=k$, perturbed by a term in the action of the form 24, 26] $\delta S=$ $\left(\lambda / k^{2}\right) \int d^{2} z \phi_{a b}(z, \bar{z}) J^{a}(z) \bar{J}^{b}(\bar{z})$, where $\lambda=f^{-2}-k$. Here $\phi_{a b}$ is the KM primary field in the adjoint representation of $\operatorname{psl}(2 \mid 2), J^{a}$ and $\bar{J}^{b}$ are the left/right chiral components of the $\operatorname{psl}(2 \mid 2)$ Noether currents [24], and $\lambda$ parametrizes the line of fixed points, mentioned above.

The conjectured link [5] between the WZ model and the IQH transition can be formulated through the notion of the point contact conductance (PCC) 27]. The PCC is a statistically fluctuating quantity. (i) On one hand, the scaling dimension $X_{q}$ of the $q$-th moment of the PCC at the IQH transition has been proven to be simply related to the exponent $\Delta_{q}$ [13, 14, $X_{q}=2 \Delta_{q}(|q|<1 / 2)$. (ii) On the other hand, the scaling dimension $x_{q}$ of the operator in the $W Z$ model carrying the same representation of the global psl(2|2) symmetry as the $q$-th moment of the PCC in the CCNM (possessing the same $\operatorname{psl}(2 \mid 2)$ symmetry), was conjectured [5] to be a quadratic func- 
tion of $q$. (iii) If one combines (i) and (ii), and if one assumes $X_{q}=x_{q}$ (following the conjectured description of the IQH transition by the WZ model), then the wave function exponents $\Delta_{q}$ at the IQH transition would be quadratic functions of $q$, as in Eq. (3).

As already mentioned, this WZ theory is known to describe transport properties of the chiral unitary class AIII [17, 18, 19], lacking time-reversal symmetry. Below we demonstrate the correctness of the conjecture made in item (ii) above, at a boundary, and for the time-reversal invariant version of the AIII model, the chiral orthogonal class BDI 17, 20]. Just as its cousin with broken timereversal symmetry, the chiral orthogonal theory also possesses a line of fixed points. Transport properties along this line can be described [18] by the perturbation of the KM point of the $\operatorname{psl}(2 \mid 2)$-invariant WZ theory described above, when the field $\phi_{a b}$ is replaced by the Kronecker delta, $\phi_{a b} \rightarrow \delta_{a b}$. Denote the corresponding coupling constant by $\lambda_{t}$. Consider the theory in the upper half plane where the system simply ends at the boundary, and an operator of scaling dimension (a 'conformal weight') $x_{\rho}^{s}\left(\lambda_{t}\right)$ on the boundary. At the KM point, where the perturbation vanishes, $\lambda_{t}=0$, such an operator is described by a representation $\rho$ of the global $\operatorname{psl}(2 \mid 2)$ symmetry. It is known 24] that $x_{\rho}^{s}\left(\lambda_{t}=0\right)=C_{\rho}^{(2)} / k$, where $C_{\rho}^{(2)}$ is the quadratic Casimir invariant in the representation $\rho$. It turns out to be straightforward 28 to compute the change of the scaling dimension, order by order in the bulk coupling constant $\lambda_{t}$, yielding a geometric series. The result is simply $x_{\rho}^{s}\left(\lambda_{t}\right)=C_{\rho}^{(2)} /\left(k+\lambda_{t}\right)$. Note that for the (continuous series) representation $\rho$ of $\operatorname{psl}(2 \mid 2)$ in which the $q$-th moment of the PCC at the IQH transition transforms, one has $C_{\rho}^{(2)}=q(1-q)$. This proves our claim that the spectrum of scaling dimensions $x_{\rho}^{s} \rightarrow x_{q}^{s}$ of corresponding boundary operators in symmetry class BDI is a strictly quadratic function of $q$.

In summary, our numerical results clearly demonstrate that both, the boundary and the bulk MF spectra, $\Delta_{q}^{s}$ and $\Delta_{q}^{b}$, significantly deviate from parabolicity, and that their $q$-dependent ratio is significantly different from 2 . (These conclusions were recently also reached, independently, by Evers, Mildenberger, and Mirlin [29].) These results for the bulk as well as the boundary MF spectra impose important constraints on any analytical theory for the IQH plateau transition. Furthermore, we have demonstrated analytically exact parabolicity of related boundary spectra in the 2D chiral orthogonal 'GadeWegner' symmetry class BDI.

We thank F. Evers, A. Mildenberger, and A. D. Mirlin for helpful discussions and for sharing their data prior to publication. We are grateful to T. Ohtsuki for his helpful suggestions on numerical algorithm. This work was partly supported by the Next Generation Super Computing Project, Nanoscience Program and a Grant-inAid for Scientific Research (No. 16GS0219) from MEXT,
Japan (HO and AF), NSF Career award DMR-0448820, NSF MRSEC DMR-0213745 (IAG), and DMR- 0706140 (AWWL). Numerical calculations were performed on the RIKEN Super Combined Cluster System.

* Present address: Department of Physics, Kyoto University, Sakyo-ku, Kyoto 606-8502, Japan

[1] K. v. Klitzing, G. Dorda, and M. Pepper, Phys. Rev. Lett. 45, 494 (1980).

[2] R. E. Prange and S. M. Girvin (eds.), The quantum Hall effect (Springer-Verlag, New York, 1990).

[3] H. Levine, S. B. Libby, and A. M. M. Pruisken, Phys. Rev. Lett. 51, 1915 (1983); D. E. Khmelnitskii, JETP Lett. 38, 552 (1983).

[4] H. Obuse et al., Phys. Rev. Lett. 98, 156802 (2007); Physica E 40, 1404 (2008).

[5] M. R. Zirnbauer, arXiv:hep-th/9905054.

[6] M. J. Bhaseen et al., Nucl. Phys. B580, 688 (2000); A. M. Tsvelik, Phys. Rev. B 75, 184201 (2007).

[7] A. LeClair, arXiv:0710.3778v1.

[8] M. Bershadsky, S. Zhukov, and A. Vaintrob, Nucl. Phys. B559, 205 (1999); N. Berkovits, C. Vafa, and E. Witten, J. High Energy Phys. 03 (1999) 018.

[9] F. Evers and A. D. Mirlin, arXiv:0707.4378.

[10] F. Wegner, Z. Phys. B 36, 209 (1980); B. Duplantier and A. W. W. Ludwig, Phys. Rev. Lett. 66, 247 (1991).

[11] A. R. Subramaniam et al., Phys. Rev. Lett. 96, 126802 (2006).

[12] A. D. Mirlin et al., Phys. Rev. Lett. 97, 046803 (2006).

[13] F. Evers, A. Mildenberger, and A. D. Mirlin, Phys. Rev. B 64, 241303(R) (2001).

[14] R. Klesse and M. R. Zirnbauer, Phys. Rev. Lett. 86, 2094 (2001).

[15] A. W. W. Ludwig et al., Phys. Rev. B 50, 7526 (1994).

[16] C. Mudry, C. Chamon, and X.-G. Wen, Nucl. Phys. B466, 383 (1996).

[17] R. Gade and F. Wegner, Nucl. Phys. B360, 213 (1991); R. Gade, Nucl. Phys. B398, 499 (1993).

[18] S. Guruswamy, A. LeClair, and A. W. W. Ludwig, Nucl. Phys. B583, 475 (2000).

[19] M. Bocquet and J. T. Chalker, Phys. Rev. B 67, 054204 (2003).

[20] M. R. Zirnbauer, J. Math. Phys. 37, 4986 (1996).

[21] A. Altland and M. R. Zirnbauer, Phys. Rev. B 55, 1142 (1997).

[22] J. T. Chalker and P. D. Coddington, J. Phys. C 21, 2665 (1988).

[23] J. Cardy, Nucl. Phys. B240, 514 (1984).

[24] V. G. Knizhnik and A. B. Zamolodchikov, Nucl. Phys. B247, 83 (1984).

[25] For a recent account, see: G. Götz, T. Quella, and V. Schomerus, J. High Energy Phys. 03 (2007) 003.

[26] T. Quella, V. Schomerus, and T. Creutzig, arXiv:0712.3549.

[27] M. Janssen, M. Metzler, and M. R. Zirnbauer, Phys. Rev. B 59, 15836 (1999).

[28] The simplicity of this kind of perturbation expansion in a related context was observed in Ref. [26.

[29] F. Evers, A. Mildenberger, and A. D. Mirlin, Phys. Rev. Lett. 101, 116803 (2008). 